\title{
Transcutaneous electrical nerve stimulation and placebo analgesia: is the effect the same for young and older individuals?
}

This article was published in the following Dove Press journal:

Clinical Interventions in Aging

\author{
Inès Daguet ${ }^{1,2}$ \\ Kayla Bergeron-Vézina ${ }^{1,2}$ \\ Marie-Philippe Harvey ${ }^{1,2}$ \\ Marylie Martel ${ }^{1,2}$ \\ Guillaume Léonard 2,3
}

'Faculté de médecine et des sciences de la santé, Université de Sherbrooke, Sherbrooke, QC, Canada; ${ }^{2}$ Research

Center on Aging, Institute of Geriatrics of Sherbrooke, Sherbrooke, QC, Canada; ${ }^{3}$ École de réadaptation, Faculté de médecine et des sciences de la santé, Université de Sherbrooke, Sherbrooke, QC, Canada
Correspondence: Guillaume Léonard Centre de recherche sur le vieillissement, CIUSSS de l'Estrie-CHUS, 1036, rue Belvédère Sud, Sherbrooke, QC JIH 4C4, Canada Tel + I 8198206868 ext 45246

$\mathrm{Fax}+\mathrm{I} 8198206864$

Email guillaume.leonard2@ usherbrooke.ca
Purpose: Placebo analgesia refers to a perceived reduction in pain intensity following the administration of a simulated or otherwise medically ineffective treatment. Previous studies have shown that many factors can influence the magnitude of placebo analgesia. However, few investigations have examined the effect of age on placebo analgesia, and none have done it in the context of electrotherapeutic interventions. The objective of this study is to compare the placebo response induced by sham transcutaneous electrical nerve stimulation (TENS) between young and older individuals, using an experimental heat-pain paradigm.

Patients and methods: Twenty-two young (21-39 years) and 22 older (58-76 years) healthy adults participated in this comparative study. Experimental heat pain was evoked with a thermode (2-min stimulation at a constant individually adjusted temperature) applied on the lumbar region. Participants were asked to evaluate the intensity of their pain using a computerized visual analog scale. Experimental pain was induced before and after an unconditioned placebo intervention (placebo TENS) applied for $25 \mathrm{~min}$.

Results: In young individuals, no significant pain reductions were noted, whereas in older individuals, a statistically significant pain reduction was observed after the placebo stimulation $(P<0.01)$. Between-group analyses revealed that placebo analgesia was greater in older individuals ( $40 \%$ pain reduction) compared with young individuals ( $15 \%$ pain reduction) $(P<0.05)$. However, sham TENS increased heat-pain thresholds in the young group $(P<0.01)$, but not in the older group $(P=0.43)$.

Conclusion: Our results indicate that placebo analgesia is influenced by age, with older individuals showing larger placebo analgesia than young adults. Although these results should be confirmed in clinical pain populations, the current observations bear potentially important consequences for the design of future placebo-controlled trials and for healthcare professionals working with elderly patients.

Keywords: pain, ageing, elderly, sham treatment, placebo, rehabilitation, electrotherapy

\section{Plain language summary}

Simulated medical interventions (commonly referred to as placebo treatments) have been shown to reduce pain in many individuals. The magnitude of pain reduction following the administration of a placebo treatment is affected by many factors. The objective of this study was to determine whether age influences the magnitude of placebo analgesia. To do this, we compared the effect of a simulated treatment commonly used in rehabilitation (transcutaneous electrical nerve stimulation or TENS) on pain between young and elderly individuals. We observed that the magnitude of the placebo response was greater in older individuals, compared to young individuals, suggesting that age is a key factor that can affect the magnitude of the placebo effect. 


\section{Introduction}

Placebo analgesia refers to a perceived reduction in pain following the administration of a simulated or otherwise medically inactive treatment. ${ }^{1}$ Placebo analgesia has been observed in healthy individuals and chronic pain patients. ${ }^{2,3}$ Placebo effects can have diverse consequences on health problems in medical practice and clinical research. ${ }^{4}$ The magnitude of placebo analgesia is known to be influenced by numerous factors, including conditioning,${ }^{5,6}$ emotions, ${ }^{7}$ motivation, ${ }^{8}$ verbal suggestions, ${ }^{9}$ social observation, ${ }^{10}$ prior experience, ${ }^{2,11}$ and previous treatments. ${ }^{3}$

The mechanisms involved in placebo analgesia are still incompletely understood, but are believed to rely on the activation of endogenous pain-modulation systems. ${ }^{12,13}$ Past observations have suggested that the efficacy of these endogenous pain-modulation systems decreases with age,${ }^{14-16}$ raising the possibility that older individuals could show reduced placebo responses, compared with young individuals. ${ }^{17}$

To date, few investigators have studied the effect of age on placebo analgesia and those who did have reported controversial results. ${ }^{18-20}$ For instance, Lasagna et al observed in a group of postoperative patients that placebo responders were, on average, older than placebo non-responders. ${ }^{18}$ This observation suggests that the analgesic effect of a placebo intervention (in this case, the administration of a simulated analgesic solution via perfusion) is greater in elderly individuals. This interpretation was challenged by the results of Ho et al, who observed that the pain-inhibitory effect of a placebo pill was higher in young patients compared with older patients in a population of individuals suffering from migraine. ${ }^{19}$ These inconsistencies could be due to different routes of administration (injection versus pill) or the pain population studied (type of pain). ${ }^{21}$ To our knowledge, no studies have investigated the effect of age on the placebo effect triggered by electrotherapeutic interventions.

The aim of the present study was to compare the magnitude of placebo analgesia between a group of young and a group of older individuals using an experimental heat-pain paradigm and an unconditioned placebo electrotherapeutic intervention (sham transcutaneous electrical nerve stimulation [TENS]). We hypothesized that young individuals would show higher placebo analgesic responses than older individuals after the placebo TENS intervention.

\section{Patients and methods}

\section{Participants}

Twenty-two young (21-39 years) and 22 older (58-76 years) healthy adults were included in the study. For safety reasons, individuals with pacemakers or metal implants were excluded, as were pregnant women. People suffering from an existing neurological or pain condition were also excluded. Individuals with prior experience with TENS treatments were not included in this investigation. Participants were asked to refrain from consuming caffeine and tobacco products 3 hours before and short-term analgesics (for acute pain such as headache, fever, etc.) 6 hours before testing. The experiment took place at the Research Center on Aging of the Centre intégré universitaire de santé et de services sociaux (CIUSSS) de l'Estrie-CHUS between November 2012 and May 2016. Participants were all French-speaking community-dwelling individuals. The study was approved by the CIUSSS de l'Estrie Research Ethics Committee (Approval \# 21-01-2016), and each participant provided written informed consent before participating in the study.

\section{Pain evaluations}

Participants assessed their pain perception using a computerized visual analog scale (CoVAS; Medoc Advanced Medical Systems, Ramat Yishai, Israel) ranging from 0 (no pain) to 100 (intolerable pain) during tonic heat-pain tests performed before and after the placebo intervention. This method of pain evaluation has been validated and has a good reliability. ${ }^{22-24}$

\section{Tonic heat-pain test}

At the beginning of the experimental session, a pretest was conducted to familiarize participants with the CoVAS and the Peltier-type thermode $(30 \times 30 \mathrm{~mm}$, TSA-II, Medoc advanced Medical Systems) used to induce experimental pain. During the pretest, the thermode was placed on the lower thoracic region of the back of the participants. Thermode temperature gradually rose from $32^{\circ} \mathrm{C}$ to $51^{\circ} \mathrm{C}$ (rising rate $=0.3^{\circ} \mathrm{C} / \mathrm{sec}$ ). Participants used the CoVAS to assess their pain during the thermode stimulation. They were instructed to start moving the cursor of the CoVAS when they started to feel pain (pain perception threshold) and that the cursor needed to be at " 100 " mark when pain was unbearable (100/100; pain tolerance threshold). This procedure was repeated until the pain reports were consistent between trials $\left(\leq 1^{\circ} \mathrm{C}\right.$ difference for pain perception and pain tolerance thresholds).

The tonic heat pain test (thermal temporal summation paradigm) consisted in the application of the thermode on the lumbar region at a constant temperature (individually adapted) for $2 \mathrm{~min} .{ }^{25}$ Participants were told that the thermode temperature could rise, remain stable, or decrease during 
the test. In fact, after a constant rise $\left(1^{\circ} \mathrm{C} / \mathrm{sec}\right)$ from baseline $\left(32^{\circ} \mathrm{C}\right)$ to the individually predetermined temperature, the temperature of the thermode remained constant throughout the whole 2-min heat-pain test. Mentioning that the temperature could rise, remain stable, or decrease during the heat pain test ensured that participants provide a timely accurate evaluation of their pain, without creating expectations with regard to the nociceptive stimulus, and influencing pain assessment (see Tousignant-Laflamme et $\mathrm{al}^{25}$ for a similar approach). The short-term (within-day) and long-term (between days) test-retest reliability of individualized thermal temporal summation paradigms is well established. ${ }^{26}$

The temperature used during the heat-pain test was determined during the pretest (temperature rated at 50/100 with the CoVAS). ${ }^{25,27}$ Participants assessed their pain with the CoVAS during the 2-min test. Mean pain intensity ratings obtained during the 2-min heat-pain test were calculated and used in subsequent analyses. The heat-pain test was performed on 5 occasions, always using the same individually adapted thermode temperature: 1) at baseline (T0), 2) during the placebo intervention (T1; after 15 min of sham TENS), 3) immediately after the placebo intervention (T2; at the end of the 25 min of sham TENS), 4) 15 min after the placebo TENS intervention (T3), and 5) $30 \mathrm{~min}$ after the placebo TENS intervention (T4). To avoid pain sensitization, the thermode was applied on adjacent regions of the lumbar region, and never on the exact same site.

\section{Heat-pain threshold}

Heat-pain thresholds (HPTs) were evaluated on the lumbar region with the thermode, using the method of limits. ${ }^{22,28}$ Participants were advised that the temperature of the thermode would gradually increase and that they would need to report their first pain sensation by clicking the left button of a computer mouse (baseline $=32^{\circ} \mathrm{C}$; rising rate $=0.3^{\circ} \mathrm{C} / \mathrm{sec}$ ) HPTs were measured at baseline (T0), during the placebo intervention (T1; after 15 min of sham TENS), and immediately after the placebo intervention (T2). A total of 3 HPTs values were taken at each time measure and then averaged to obtain a single HPT value.

\section{Placebo intervention}

The placebo intervention was a sham TENS intervention that was delivered using 2 pairs of rubber silicone electrodes connected to a digital Eclipse Plus apparatus (Empi, St Paul, MN, USA), applied for $25 \mathrm{~min}$. Electrodes were placed on the lower thoracic and lumbar regions. The frequency was set at $100 \mathrm{~Hz}$ and the pulse duration at $60 \mu \mathrm{s}$. However, the TENS apparatus was inactivated using a hidden device, which disabled the electrodes (electric stimulation was applied to built-in resistors) without changing the TENS equipment display. The participants were told that there was an electric current (indication of stimulation on the TENS device). In reality, the electric current was dissipated in the resistors, and no electrical stimulation was given to the participants.

\section{Expectations of pain modification}

The participants communicated their expectations 2 min before the application of sham TENS (before the first heatpain test [T0]) and 2 min after the start of the sham TENS stimulation (before the second heat-pain test [T1]). Before the stimulation, these expectations were reported to the experimenter, whereas during the placebo TENS intervention, the therapist assessed the expectations of the participants. Volunteers were asked to evaluate the effect they expected the TENS intervention would have on pain intensity (increase, no change, or decrease). The consent form specified that TENS is an electrotherapeutic modality commonly used to stimulate peripheral nerves and reduce pain. Participants were also informed that the aim of the study was to evaluate the effect of a specific type of TENS treatment on pain. Participants did not receive any further guidance with regard to the expected effect of TENS intervention. Subsequently, participants had to estimate a percentage of expected pain variation $(0 \%=$ no change; $+100 \%=$ total pain decrease; $-100 \%=$ strong pain increase). Expectations were gathered for 30 of the 44 participants (14 young and 16 older adults).

\section{Sample size and statistical analysis}

To facilitate comparisons, and because pain ratings were obtained continuously during the 2 -min tonic heat-pain test (CoVAS sampling rate $=10 \mathrm{~Hz}$ ), the pain intensity ratings obtained during each 2-min tonic heat-pain test were averaged for each participant and time point, and the mean was used in subsequent analyses. The amplitude of the placebo effect ( $\%$ of change in pain) was calculated using the following formula: ([T0 pain intensity - T2 pain intensity]/T0 pain intensity) $\times 100$. The study was designed to detect a betweengroup difference of 20 points on the CoVAS (clinically important difference). ${ }^{29}$ To detect this difference, with $80 \%$ statistical power and a 5\% significance level, we determined that 22 young and 22 older adults should be enrolled in the study (estimated SD of 23, based on preliminary results).

Given the small number of participants and because visual inspection of the histograms (frequency distribution) did not allow us to assume that the data were normally distributed, 
nonparametric tests were used. Friedman tests and Wilcoxon signed-rank tests were used to compare the pain intensity scores and the HPTs before, during, and after the placebo intervention (within-subject analysis). This allowed us to determine whether the sham TENS treatment influenced pain perception. MannWhitney tests were used to compare the pain intensity scores, HPT, and the magnitude of placebo analgesia (\% change in pain) between the young and the older groups (between subject analyses). Statistical differences were considered significant if $P<0.05$ was obtained. All tests were performed using SPSS (version 19.0 for Windows, Chicago, IL, USA).

\section{Results}

\section{Characteristics of the participants}

Of the 50 participants who reported interest to take part in the study, 49 met the inclusion criteria (Figure 1). Five participants withdrew before the intervention started for personal reasons. Twenty-two young participants aged 21-39 years (mean: 27; 11 men and 11 women) and 22 older individuals aged 58-76 years (mean: 67; 11 men and 11 women) completed the study. Stimulation temperatures for the heat-pain tests at baseline were comparable between young $\left(45.9^{\circ} \mathrm{C} \pm 0.5^{\circ} \mathrm{C}\right)$ and older participants $\left(46.5^{\circ} \mathrm{C} \pm 0.4^{\circ} \mathrm{C} ; P=0.53\right)$.

\section{Pain intensity during heat-pain tests}

The average pain intensity scores obtained for the heat-pain tests before, during, and after the placebo intervention are presented in Figure 2. Every participant experienced pain before placebo TENS application (T0; all pain intensity scores $>17$ ). Mann-Whitney tests showed that mean pain intensity ratings obtained during the tonic heat-pain test at baseline (T0) did not differ between the young (42.4 \pm 2.7$)$ and the older groups (41.0 $\pm 3.2 ; P=0.97)$.

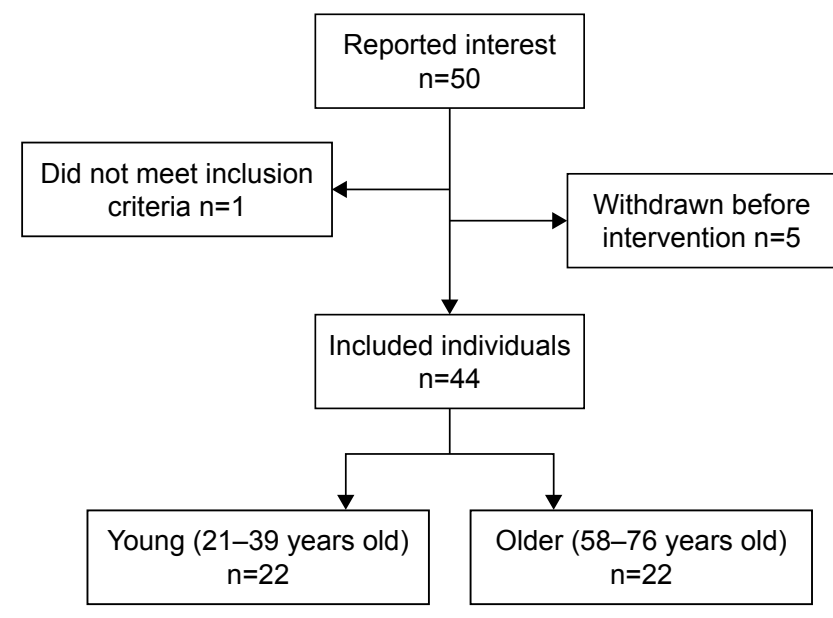

Figure I Flow chart for study participant enrollment.

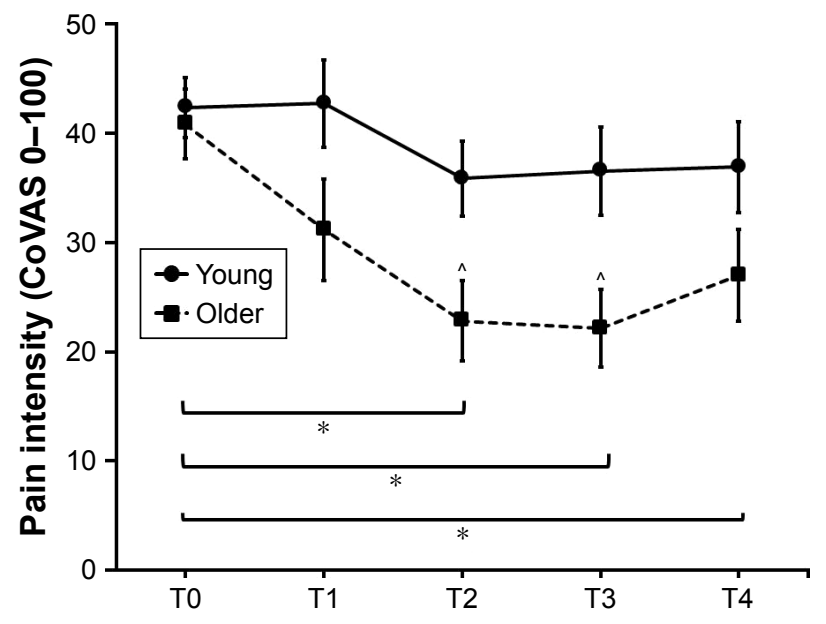

Figure 2 Mean $( \pm S E M)$ pain intensity before $(T 0)$, during (TI), immediately after $(T 2)$, 15 min after (T3), and 30 min after (T4) sham TENS.

Notes: $* P<0.05$ for pain intensity in the older group during $(\mathrm{TI})$ and after the sham TENS application (T2, T3 and T4) compared to baseline (T0). ${ }^{\wedge P}<0.05$ for the difference in pain scores at $\mathrm{T} 2$ and $\mathrm{T} 3$ between the young and the older groups. Abbreviations: CoVAS, computerized visual analog scale; SEM, standard error of the mean; TENS, transcutaneous electrical nerve stimulation.

Pain intensity tended to decrease after the sham TENS stimulation in young and older adults. However, no significant pain reduction was observed at T1, T2, T3, and T4 in the young group, when compared with baseline (T0; all $\left.P_{\mathrm{s}}>0.05\right)$. As depicted in Figure 2, the pain reductions were of greater magnitude in the older group. This was confirmed by the Friedman test, which revealed a statistically significant difference in pain in the older group $(P<0.01)$. Wilcoxon signed-rank tests further revealed statistically significant reductions in pain scores in the older group after the sham TENS application (T2, T3, and T4) when compared with baseline (T0; all $\left.P_{\mathrm{s}}<0.01\right)$. Pain reduction during sham TENS application (T1) did not reach statistical significance $(P=0.07)$.

Mann-Whitney tests were also used to directly compare the pain scores during and after TENS application between the young and the old groups. Results showed that the pain intensity ratings were significantly lower in the older group immediately after (T2) and 15 min after (T3) TENS stimulation, compared with the young group (all $P \mathrm{~S} \leq 0.01$ ), once again suggesting a greater placebo effect in the older group. Pain evaluations were comparable between the groups during TENS (T1), as well as $30 \mathrm{~min}$ after the end of the placebo intervention (T4; all $P_{\mathrm{S}}>0.05$ ).

\section{Magnitude of placebo analgesia}

The magnitude of placebo analgesia (\% change in pain) in the young and the older groups is shown in Figure 3. As it can be seen from the figure, the amplitude of the placebo effect was larger in the older group than in the young group, both 


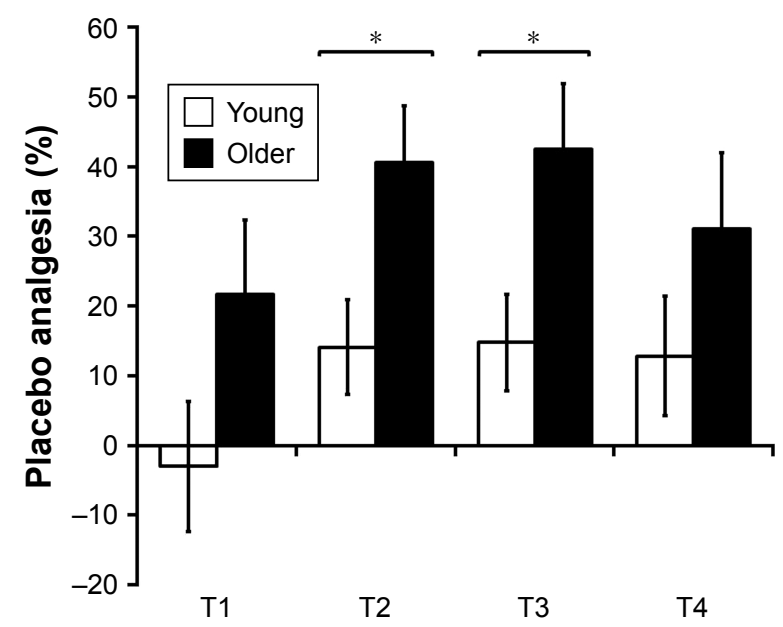

Figure 3 Mean $( \pm S E M)$ placebo analgesia (\% change in pain intensity) in the young group $(n=22)$ and the older group $(n=22)$ during $(T I)$, immediately after $(T 2), 15 \mathrm{~min}$ after (T3), and 30 min after (T4) sham TENS. $* P<0.05$ the difference between young and older groups.

Abbreviations: SEM, standard error of the mean; TENS, transcutaneous electrical nerve stimulation.

immediately after $(\mathrm{T} 2 ; P<0.05)$ and $15 \mathrm{~min}$ after $(\mathrm{T} 3 ; P<0.05)$ the sham TENS intervention. Differences in placebo effects during $(\mathrm{T} 1 ; P=0.15)$ and 30 min after $(\mathrm{T} 4 ; P=0.09)$ sham TENS stimulation failed to reach statistical significance.

\section{Heat-pain threshold}

HPTs obtained before, during, and after the placebo intervention are presented in Table 1. Baseline HPTs were comparable between groups $(P=0.92)$. Friedman tests revealed that sham TENS increased HPT in the young group $(P<0.01)$, but not in the older group $(P=0.43)$. In the young group, the HPTs increase reached statistical significance during (T1) and after (T2) the placebo intervention when compared with baseline (all $P<0.05$ ).

\section{Expectations of pain modification}

Seven ( 5 in the young and 2 in the older groups) of the 44 participants identified the presence of a placebo treatment (success rate of blinding 84\%). Before the placebo TENS stimulation, young adults expected an average pain reduction

Table I Mean ( \pm SEM) heat-pain thresholds before, during, and after sham TENS stimulation

\begin{tabular}{lll}
\hline $\begin{array}{l}\text { Heat-pain } \\
\text { thresholds }\left({ }^{\circ} \mathbf{C}\right)\end{array}$ & $\begin{array}{l}\text { Young } \\
(\mathbf{n}=\mathbf{2 2})\end{array}$ & $\begin{array}{l}\text { Older } \\
(\mathbf{n}=\mathbf{2 2})\end{array}$ \\
\hline T0 - before & $42.7( \pm 0.6)$ & $42.7( \pm 0.6)$ \\
TI - during & $43.2( \pm 0.6)^{*}$ & $43.1( \pm 0.6)$ \\
T2 - after & $43.5( \pm 0.6)^{*}$ & $42.4( \pm 1.0)$ \\
\hline
\end{tabular}

Note: $* P<0.05$ for the difference between before, during, and after placebo TENS stimulation.

Abbreviations: SEM, standard error of the mean; TENS, transcutaneous electrical nerve stimulation.

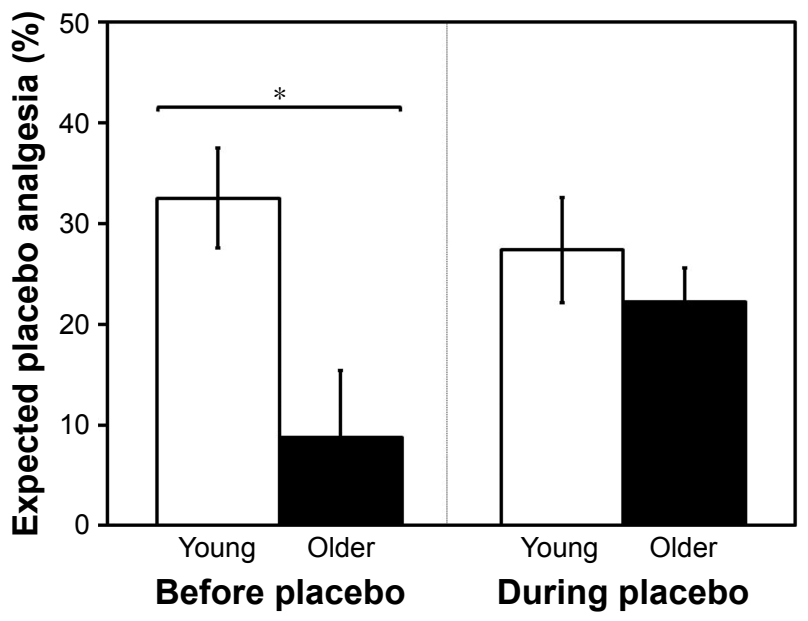

Figure 4 Mean $( \pm$ SEM) expectations for pain variations before and during sham TENS stimulation in the young $(n=14)$ and the older group $(n=16) . * P<0.05$ the difference between young and older groups.

Abbreviations: SEM, standard error of the mean; TENS, transcutaneous electrical nerve stimulation.

of $33 \% \pm 19 \%$, whereas older individuals expected a pain reduction of $9 \% \pm 27 \%$ ( $P<0.05$; see Figure 4$)$. However, during the placebo TENS intervention, expectations were around $25 \%$ of pain reduction in both groups $(P=0.75)$. Hence, in the older group, expectations were higher during the treatment than before the intervention $(P<0.05)$, whereas expectations did not change significantly in the young group $(P=0.17)$.

\section{Discussion}

The objective of this study was to better understand the effects of age on the placebo analgesia induced by an electrotherapeutic intervention. Contrary to our initial hypothesis, we observed that the placebo response was stronger in older than in young individuals. This difference was considerable, with pain reductions being more than twice as important in the older group. These observations are in line with the results of Lasagna et al who reported in a group of postoperative patients, that placebo responders tended to be older than placebo non-responders. ${ }^{18}$ However, these results are in opposition with the results of Ho et $\mathrm{al}^{19}$ and Wrobel et al..$^{20}$ These discrepancies could be due to the characteristics of the placebo treatment administered (pharmacological versus non-pharmacological interventions), the population studied (acute, chronic/recurrent pain, or experimental pain), and the paradigm used (presence/absence of a conditioning phase). ${ }^{8}$

\section{Effect of placebo TENS on HPTs}

Our results show a significant, although relatively small $\left(<1.0^{\circ} \mathrm{C}\right)$, increase in HPTs after placebo TENS stimulation in the young group, suggesting a hypoalgesic placebo effect. 
Previous studies have shown comparable pain thresholds increments after the administration of placebo treatments in young individuals. ${ }^{30,31}$ Increments in HPT of $\sim 1.0^{\circ} \mathrm{C}$ were observed after intravenous administration of low doses of a potent opioid analgesic drug (remifentanil $0.05 \mu \mathrm{g} / \mathrm{kg} / \mathrm{min}$ ), ${ }^{32}$ suggesting that even small increases in HPT can be clinically significant. However, in the absence of specific values defining a clinically significant change for HPT, it is difficult to determine if the changes noted in this study are of clinical importance. Surprisingly, in the older group, HPTs did not change after the placebo TENS stimulation. This absence of significant change could partly be explained by the high variability of HPT responses after the placebo intervention in the older group. This high variability has been reported in elderly individuals for other types of measures. ${ }^{33}$

\section{Psychological and neurophysiological factors}

Age differences in placebo analgesia effectiveness could be attributable to psychological factors. ${ }^{34}$ Numerous studies have shown that psychological factors, such as conditioning, ,,6 emotions, ${ }^{7}$ motivation, ${ }^{8}$ and previous experience, ${ }^{10,11}$ can influence the magnitude of placebo analgesia. Some evidence suggests that expectation and conditioning are 2 of the most influential factors, with high expectations or a high conditioning effect being associated with greater placebo effects. Some researchers have suggested that conditioning and expectations are intrinsically interconnected. ${ }^{35}$ Conditioning is, in fact, often seen as a potent and effective way to increase the expectations related to a given intervention. ${ }^{5,6}$ Investigators supporting this interpretation showed that previous experience influences both expectations and the magnitude of placebo analgesia., ${ }^{2,35}$ In that sense, the larger placebo effect noted in elderly individuals could potentially be explained by the fact that older individuals have received a greater number of effective treatments during their lifetime, which acted as positive conditioning procedures.

If this holds true, older individuals would be expected to have higher expectations. On the contrary, we observed that older individuals had lower expectations with regard to sham TENS before application. Surprisingly, the expectations of older individuals significantly increased once the sham intervention was initiated. It remains uncertain why the expectations of the participants of the older group changed over time. Perhaps older individuals became more confident with regard to the intervention when they were taken in charge by the therapist and saw the TENS apparatus. Future studies are required to test this hypothesis and determine if factors related to the therapist and/or the intervention can differentially influence the expectations of young and older individuals.

Age differences in the magnitude of placebo analgesia could also be explained by neurophysiological factors. Indeed, placebo analgesia has been thought to rely on the activation of descending pain-modulating mechanisms ${ }^{12,13}$ and these endogenous pain-modulation systems have been shown to be less efficient in elderly individuals. ${ }^{14-16}$ In this case, the presence of a defective top-down circuitry suggests that other mechanisms (eg, top-top modulation) could step in. For instance, a functional compensatory mechanism, named the posterior-anterior shift in aging, has been observed in older adults. ${ }^{36-39}$ These studies, which compared brain activity measures between young and older adults, have reported increased activity in the prefrontal cortex and decreased activity in the occipital cortex in older individuals during working memory and visual attention tasks. ${ }^{36,37}$ Similar cerebral mechanisms could be triggered in the context of a therapeutic treatment and may potentially explain why elderly individuals show preserved/increased placebo response, despite defective top-down circuitry.

\section{Limitations}

The tonic heat-pain paradigm that was used in this study is a well-validated model, but does not reflect clinical pain situations. Indeed, the mechanisms involved in placebo analgesia may vary between healthy individuals and patients suffering from chronic pain. Reproducing these results in clinical pain populations is warranted before any final conclusion can be made.

When participants were asked at the end of the testing session if they thought that the TENS stimulation was effective, 7 of them (16\%) identified the presence of a sham condition. However, most participants (84\%) did not comment on sham or placebo, suggesting that blinding was generally preserved. Moreover, it should be noted that expectations with regard to the effectiveness of sham TENS were not gathered for the first 14 of the 44 participants. Nevertheless, expectations were evaluated in a similar number of young $(n=14)$ and older ( $\mathrm{n}=16)$ adults, and it is unlikely that the missing values would have affected the interpretation of our results.

Finally, it should be noted that previous pain experiences (including prior analgesic efficacy, medical conditions, stressful life events, coping strategies, and other psychological characteristics such as anxiety and depression) that could potentially affect placebo analgesia were not evaluated in the current study. 


\section{Conclusion}

The main point that emerges from this study is that the magnitude of placebo analgesia triggered by a common electrotherapeutic intervention (TENS) is greater in older than in young adults. This difference could be related to a difference in psychological factors, neurophysiological mechanisms, or a combination of both. Although these results still need to be confirmed in clinical pain populations, the current observations suggest that age is a factor that should be considered in clinical practice, as well as in upcoming clinical trials. Further studies are necessary to identify the psychobiological mechanisms underlying the age-related differences in placebo analgesia.

\section{Acknowledgments}

The authors would like to thank Marie-Claude Girard and Mathieu Hamel for their help with data collection, and Philippe Chalaye for his thoughtful comments on this manuscript.

\section{Author contributions}

All authors contributed to data analysis, drafting, and critically revising the paper, and agreed to be accountable for all aspects of the work.

\section{Disclosure}

G Léonard is supported by Fonds de recherche Santé (FRQ-S, Québec, Canada) and by a grant from the Natural Sciences and Engineering Research Council of Canada (NSERC). The authors report no other conflicts of interest in this work.

\section{References}

1. Benedetti F. The opposite effects of the opiate antagonist naloxone and the cholecystokinin antagonist proglumide on placebo analgesia. Pain. 1996;64(3):535-543.

2. Colloca L, Benedetti F. How prior experience shapes placebo analgesia. Pain. 2006;124(1-2):126-133.

3. Muller M, Kamping S, Benrath J, et al. Treatment history and placebo responses to experimental and clinical pain in chronic pain patients. Eur J Pain. 2016;20(9):1530-1541.

4. Holmes RD, Tiwari AK, Kennedy JL. Mechanisms of the placebo effect in pain and psychiatric disorders. Pharmacogenomics J. 2016; 16(6):491-500.

5. Amanzio M, Benedetti F. Neuropharmacological dissection of placebo analgesia: expectation-activated opioid systems versus conditioningactivated specific subsystems. J Neurosci. 1999;19(1):484-494.

6. Montgomery GH, Kirsch I. Classical conditioning and the placebo effect. Pain. 1997;72(1-2):107-113.

7. Morton DL, Watson A, El-Deredy W, Jones AK. Reproducibility of placebo analgesia: effect of dispositional optimism. Pain. 2009;146(1-2): 194-198.

8. Vase L, Robinson ME, Verne GN, Price DD. The contributions of suggestion, desire, and expectation to placebo effects in irritable bowel syndrome patients. An empirical investigation. Pain. 2003;105(1-2): $17-25$.
9. Pollo A, Amanzio M, Arslanian A, Casadio C, Maggi G, Benedetti F Response expectancies in placebo analgesia and their clinical relevance. Pain. 2001;93(1):77-84.

10. Colloca L, Benedetti F. Placebo analgesia induced by social observational learning. Pain. 2009;144(1-2):28-34.

11. Kirsch I, Kong J, Sadler P, et al. Expectancy and conditioning in placebo analgesia: separate or connected processes? Psychol Conscious (Wash D C). 2014;1(1):51-59.

12. Eippert F, Bingel U, Schoell ED, et al. Activation of the opioidergic descending pain control system underlies placebo analgesia. Neuron 2009;63(4):533-543.

13. Goffaux P, Leonard G, Marchand S, Rainville P. Placebo analgesia. In: Beaulieu P, Lussier D, Porreca F, Dickenson A, editors. Pharmacology of Pain. Seatle: IASP Press; 2010:451-473.

14. Edwards RR, Fillingim RB, Ness TJ. Age-related differences in endogenous pain modulation: a comparison of diffuse noxious inhibitory controls in healthy older and younger adults. Pain. 2003;101(1-2):155-165.

15. Washington LL, Gibson SJ, Helme RD. Age-related differences in the endogenous analgesic response to repeated cold water immersion in human volunteers. Pain. 2000;89(1):89-96.

16. Lariviere M, Goffaux P, Marchand S, Julien N. Changes in pain perception and descending inhibitory controls start at middle age in healthy adults. Clin J Pain. 2007;23(6):506-510.

17. Bingel U, Colloca L, Vase L. Mechanisms and clinical implications of the placebo effect: is there a potential for the elderly? A mini-review. Gerontology. 2011;57(4):354-363.

18. Lasagna L, Mosteller F, Von Felsinger JM, Beecher HK. A study of the placebo response. Am J Med. 1954;16(6):770-779.

19. Ho TW, Fan X, Rodgers A, Lines CR, Winner P, Shapiro RE. Age effects on placebo response rates in clinical trials of acute agents for migraine: pooled analysis of rizatriptan trials in adults. Cephalalgia. 2009; 29(7):711-718.

20. Wrobel N, Fadai T, Brassen S, Bingel U. Preserved capacity for placebo analgesia in the elderly. J Pain. 2016;17(12):1318-1324.

21. Buckalew LW, Coffield KE. An investigation of drug expectancy as a function of capsule color and size and preparation form. $J$ Clin Psychopharmacol. 1982;2(4):245-248.

22. Moloney NA, Hall TM, O'Sullivan TC, Doody CM. Reliability of thermal quantitative sensory testing of the hand in a cohort of young, healthy adults. Muscle Nerve. 2011;44(4):547-552.

23. Moloney NA, Hall TM, Doody CM. Reliability of thermal quantitative sensory testing: a systematic review. J Rehabil Res Dev. 2012;49(2): 191-207.

24. Bird ML, Callisaya ML, Cannell J, Gibbons T, Smith ST, Ahuja KDK. Accuracy, validity, and reliability of an electronic visual analog scale for pain on a touch screen tablet in healthy older adults: a clinical trial. Interact J Med Res. 2016;5(1):e3.

25. Tousignant-Laflamme Y, Page S, Goffaux P, Marchand S. An experimental model to measure excitatory and inhibitory pain mechanisms in humans. Brain Res. 2008;1230:73-79.

26. Kong JT, Johnson KA, Balise RR, Mackey S. Test-retest reliability of thermal temporal summation using an individualized protocol. J Pain. 2013;14(1):79-88.

27. Leonard G, Goffaux P, Marchand S. Deciphering the role of endogenous opioids in high-frequency TENS using low and high doses of naloxone. Pain. 2010;151(1):215-219.

28. Yarnitsky D, Sprecher E, Zaslansky R, Hemli JA. Heat pain thresholds: normative data and repeatability. Pain. 1995;60(3):329-332.

29. Farrar JT, Portenoy RK, Berlin JA, Kinman JL, Strom BL. Defining the clinically important difference in pain outcome measures. Pain. 2000;88(3):287-294.

30. Krummenacher P, Candia V, Folkers G, Schedlowski M, Schonbachler G. Prefrontal cortex modulates placebo analgesia. Pain. 2010;148(3): 368-374.

31. Martikainen IK, Hagelberg N, Mansikka H, et al. Association of striatal dopamine D2/D3 receptor binding potential with pain but not tactile sensitivity or placebo analgesia. Neurosci Lett. 2005;376(3):149-153. 
32. Kim TE, Kim KP, Shin D, et al. Assessment of the analgesic effect of remifentanil using three pain models in healthy Korean volunteers: a randomized, controlled study. Basic Clin Pharmacol Toxicol. 2012; 110(6):518-523.

33. Callisaya ML, Blizzard L, Schmidt MD, McGinley JL, Srikanth VK. Ageing and gait variability-a population-based study of older people. Age Ageing. 2010;39(2):191-197.

34. Frisaldi E, Piedimonte A, Benedetti F. Placebo and nocebo effects: a complex interplay between psychological factors and neurochemical networks. Am J Clin Hypn. 2015;57(3):267-284.

35. Benedetti F, Pollo A, Lopiano L, Lanotte M, Vighetti S, Rainero I. Conscious expectation and unconscious conditioning in analgesic, motor, and hormonal placebo/nocebo responses. J Neurosci. 2003; 23(10):4315-4323.
36. Davis SW, Dennis NA, Daselaar SM, Fleck MS, Cabeza R. Que PASA? The posterior-anterior shift in aging. Cereb Cortex. 2008;18(5): 1201-1209.

37. Cabeza R, Daselaar SM, Dolcos F, Prince SE, Budde M, Nyberg L. Task-independent and task-specific age effects on brain activity during working memory, visual attention and episodic retrieval. Cereb Cortex. 2004;14(4):364-375.

38. Grady CL, Maisog JM, Horwitz B, et al. Age-related changes in cortical blood flow activation during visual processing of faces and location. J Neurosci. 1994;14(3 Pt 2):1450-1462.

39. Madden DJ, Turkington TG, Coleman RE, Provenzale JM, DeGrado TR, Hoffman JM. Adult age differences in regional cerebral blood flow during visual world identification: evidence from H215O PET. NeuroImage. 1996;3(2):127-142.
Clinical Interventions in Aging

\section{Publish your work in this journal}

Clinical Interventions in Aging is an international, peer-reviewed journal focusing on evidence-based reports on the value or lack thereof of treatments intended to prevent or delay the onset of maladaptive correlates of aging in human beings. This journal is indexed on PubMed Central, MedLine,

\section{Dovepress}

CAS, Scopus and the Elsevier Bibliographic databases. The manuscript management system is completely online and includes a very quick and fair peer-review system, which is all easy to use. Visit http://www.dovepress. com/testimonials.php to read real quotes from published authors. 